Article

\title{
3D Photoacoustic Tomography System Based on Full-View Illumination and Ultrasound Detection
}

\author{
Mingjian Sun ${ }^{1,2, *}$, Depeng Hu ${ }^{1}$, Wenxue Zhou ${ }^{1}$, Yang Liu ${ }^{2}$, Yawei Qu ${ }^{2}$ and Liyong Ma ${ }^{1} \mathbb{D}$ \\ 1 Department of Control Science and Engineering, Harbin Institute of Technology, Weihai 264209, China; \\ wshdphit@163.com (D.H.); zwxhiter@163.com (W.Z.); maly@hitwh.edu.cn (L.M.) \\ 2 Department of Control Science and Engineering, Harbin Institute of Technology, Harbin 150000, China; \\ liuyang_6Y6@163.com (Y.L.); qyw198553@163.com (Y.Q.) \\ * Correspondence: sunmingjian@hit.edu.cn; Tel.: +86-183-6318-0812
}

Received: 25 March 2019; Accepted: 6 May 2019; Published: 9 May 2019

check for updates

\begin{abstract}
A 3D photoacoustic computed tomography (3D-PACT) system based on full-view illumination and ultrasound detection was developed and applied to 3D photoacoustic imaging of several phantoms. The system utilized an optics cage design to achieve full-view uniform laser illumination and completed 3D scanning with the rotation of a dual-element transducer (5 MHz) and the vertical motion of imaging target, which obtains the best solution in the mutual restriction relation between cost and performance. The 3D-PACT system exhibits a spatial resolution on the order of $300 \mu \mathrm{m}$, and the imaging area can be up to $52 \mathrm{~mm}$ in diameter. The transducers used in the system provides tomography imaging with large fields of view. In addition, the coplanar uniform illumination and acoustic detection configuration based on a quartz bowl greatly enhances the efficiency of laser illumination and signal detection, making it available for use on samples with irregular surfaces. Performance testing and 3D photoacoustic experiments on various phantoms verify that the system can perform 3D photoacoustic imaging on targets with complex surfaces or large sizes. In future, efforts will be made to achieve full-body 3D tomography of small animals and a multimodal 3D imaging system.
\end{abstract}

Keywords: 3D photoacoustic tomography; full-view illumination and ultrasound detection; photoacoustic coplanar; quartz bowl

\section{Introduction}

Photoacoustic imaging (PAI) is a non-invasive and non-ionized multimodal biomedical imaging method based on transient thermoelastic effects of the biological tissue. The principle is that the energy of pulsed laser light deposited in biological tissue during the process of laser absorption is converted into an ultrasonic signal which is called the photoacoustic signal through instantaneous thermoelastic expansion, and the ultrasonic transducer can receive the signal that carries information about the properties of laser absorption in biological tissue [1-7]. Through the corresponding signal processing and image reconstruction algorithms, a photoacoustic image reflecting the internal structure and function of the tissue can be obtained. Photoacoustic imaging effectively overcomes the limitations of existing pure optical imaging and pure ultrasound imaging, the contrast is based on the absorption of laser light during the photoacoustic excitation period, while the resolution is derived from the ultrasonic detection during the photoacoustic emission period. Photoacoustic imaging breaks through the limit depth of diffusion of high-resolution optical imaging (about $1 \mathrm{~mm}$ ), and uses laser-generated ultrasonic waves as a carrier to obtain optical absorption information of tissue [8,9]. It is mainly suitable for the tissues whose acoustic properties are uniform but optical properties are not. Therefore, photoacoustic imaging combines the advantages of high contrast characteristics of optical imaging 
and high penetration characteristics of ultrasonic imaging, and can provide photoacoustic images with high optical contrast and high acoustic resolution at a deeper imaging depth [10]. As a new generation of biomedical imaging technology, photoacoustic imaging can effectively realize the structure and functional imaging of biological tissue, which provides an important method for studying the morphological structure, physiological characteristics, pathological characteristics, and metabolic functions of biological tissues [11-13].

Scientific research on 3D photoacoustic imaging of small animals has become increasingly popular in recent years. There have been many 3D photoacoustic tomography (PACT) systems using different photoacoustic coupling methods, optical transmission models [14], and ultrasonic detection designs [15-20]. However, due to the limited detection angle of ultrasonic transducers, linear array PACT [16-19], spherical array PACT [20,21], arc-shaped array PACT [22], and the half-ring multispectral optoacoustic tomography (MSOT) [23] generally need to obtain complete photoacoustic signal via spatial multiplexing. In photoacoustic tomography, uniform illumination helps to obtain more comprehensive light absorption information of the imaging cross section [24]. The traditional circular scanning mainly irradiates the laser beam from the top to the bottom of the object [25-28], which results in the loss of deeper tissue structure information, while at the same time, the scattering effect of the light cannot effectively illuminate the irregular section and affects the imaging effect. From the perspective of the cost, the array ultrasonic transducer with high performance makes it not only expensive, but also a huge challenge for transducer manufacturing and data acquisition. Once formed, its scanning radius cannot be adjusted according to the size of the imaging targets, which limits the scope and flexibility of its application. There are also some photoacoustic tomography systems that acquire photoacoustic signals point by point through a single-element transducer carried by a rotary motor, whose advantages are their low cost and easy operability. The ultrasonic transducer performs a rotary motion at a predefined speed and completes the acquisition of the photoacoustic signal of the imaging section during the continuous motion process, the continuous scan of data acquisition is faster than a stop-and-go scan [29-31]. In addition, in order to improve the resolution of the photoacoustic imaging system, a negative lens can be mounted on the surface of the ultrasonic transducer to increase the receiving angle [32,33]. Due to the large field-of-view of the virtual point ultrasound transducer, some research has applied virtual point ultrasonic transducers to a photoacoustic imaging system [34-36]. Nie LM et al. developed a virtual point ultrasonic transducer with a large receiving angle and applied it to the photoacoustic tomography system. The signal-to-noise ratio of the system is greatly improved compared with the negative-lens transducers [35]. Hao F. Zhang et al. use a synthetic-aperture focusing technique based on the virtual point detector to obtain a high-resolution photoacoustic image of hemoglobin oxygen saturation in living mice [36].

In this paper, a 3D photoacoustic imaging system based on "dual-element" ultrasonic transducer is proposed under the premise of full-view imaging and uniform illumination. The system contains 1-to-8 fan-out fiber bundles and an optical cage design, which delivers approximately uniform laser radiation toward the imaging phantom and provides an exciting source for completing the photoacoustic tomography of each B-scan (B-scan refers to a cross section of photoacoustic tomography in this paper). The rotary motor carries two customized dual-foci virtual point ultrasonic transducers for photoacoustic data acquisition in the process of rotary motion, while the precise vertical stepper motor drives the imaging target to do vertical movement to complete the $3 \mathrm{D}$ scanning of it. The dual-foci virtual point ultrasonic transducer can expand the signal acceptance angle in the short focusing direction because of its large field of view, such that a relatively complete photoacoustic signal can be acquired with fewer detection units, which reduces the cost of the photoacoustic imaging system and accelerates the photoacoustic imaging speed. In the long focusing direction, the narrow sound beam and the annular illumination beam are coupled to form a photoacoustic coplanar mode, which improves the signal to noise ratio of the photoacoustic signal. The pattern of $360^{\circ}$ circular laser radiation provides uniform illumination at all angles around the imaging cross section, which facilitates photoacoustic tomography of imaging phantom with irregular surfaces. Furthermore, the 
feasibility and operability of the 3D-PACT system is validated through systematic testing and 3D photoacoustic experiments on a variety of phantoms.

\section{Methods}

\subsection{Introduction to the $3 D-P A C T$ System}

Figure 1 shows a schematic of the 3D-PACT system. The system consists of four parts, which are optical illumination, photoacoustic signal acquisition, data processing, and human computer interaction. A pulsed Nd:YAG laser source (Vigour-A-100S, Ziyu, Anshan Liaoning Province, China) with the wavelength of $532 \mathrm{~nm}$ was used for the optical illumination. The nanosecond pulsed laser with pulse repetition rate of $20 \mathrm{~Hz}$ could provide a laser beam with the pulse width of $5 \mathrm{~ns}$. The laser beam was coupled into the multimode fiber (Ceram Optec, Bonn, Germany, damage threshold was $9.1 \mathrm{~mJ} / \mathrm{mm}^{2}$ ) located in the fiber coupler after collimating and correcting the optical path. Furthermore, the multimode fiber was divided into eight branches at the output end that were evenly distributed around the water tank. After testing, the coupling ratios of the eight sub-fibers were $9.35 \%, 9.25 \%$, $9.33 \%, 9.34 \%, 9.40 \%, 9.43 \%, 9.44 \%$, and $9.41 \%$, respectively, and the total coupling ratio of the fiber was $74.95 \%$. The laser beam out of each branch passed through a convex lens and a plano-convex cylinder lens to form a rectangular strip with a thickness of about $2 \mathrm{~mm}$, as shown in Figure 2a,b, which shows the top view and the front view of the optical path, respectively. The convex lens collimates and corrects the laser beam, while the plano-convex cylinder lens focused the beam in the direction of thickness. Eight laser beams separately passed through the water tank and the transparent quartz bowl, which were placed as shown in Figure 2b, eventually converging in the middle of the tank to form a bright circular area that irradiated the surface of the sample. According to the experimental verification, the light transmittance of the water tank used in this system was about $98 \%$, which was due to the acrylic material that had excellent light transmittance.

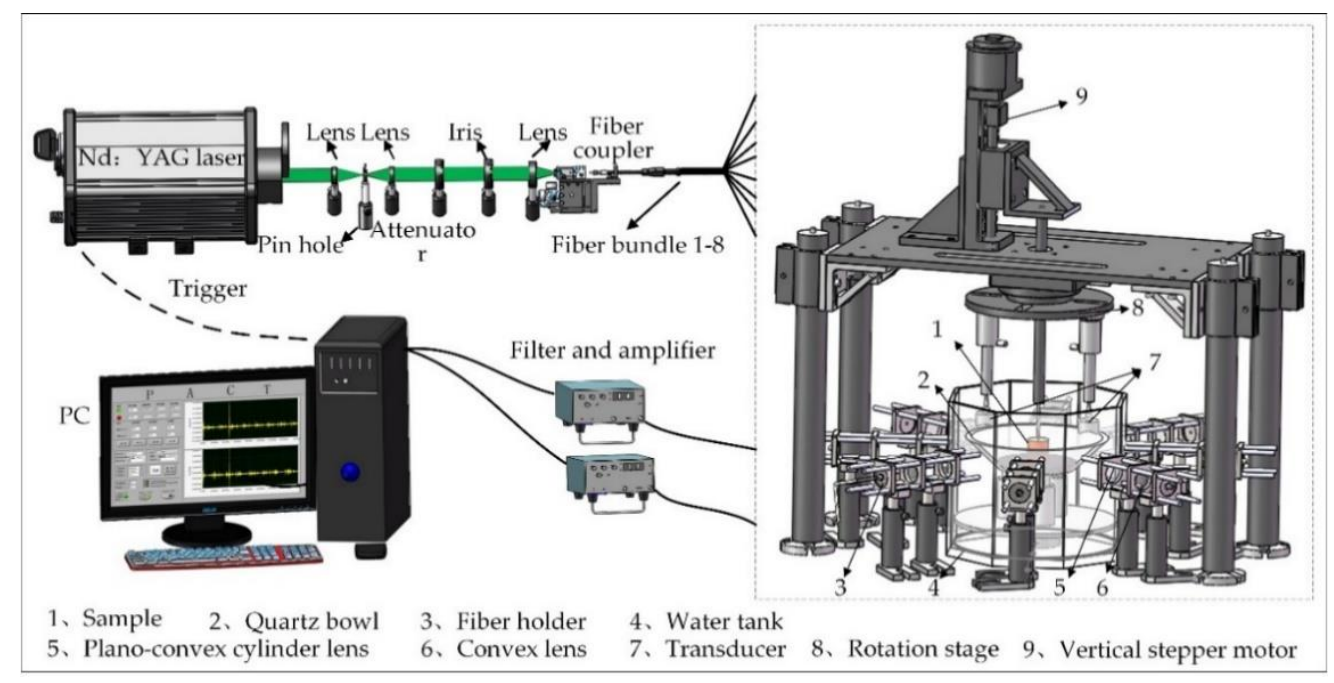

Figure 1. Schematic of the 3D-PACT system. 


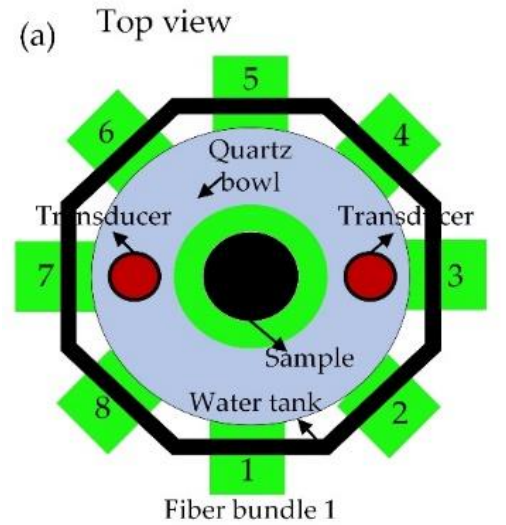

(c)

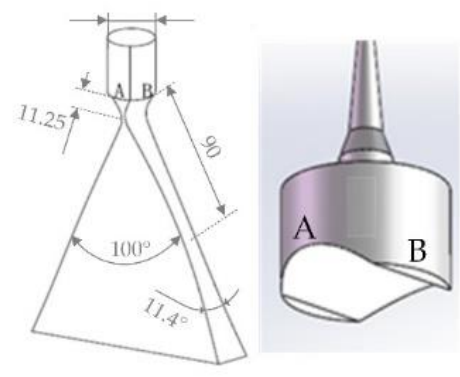

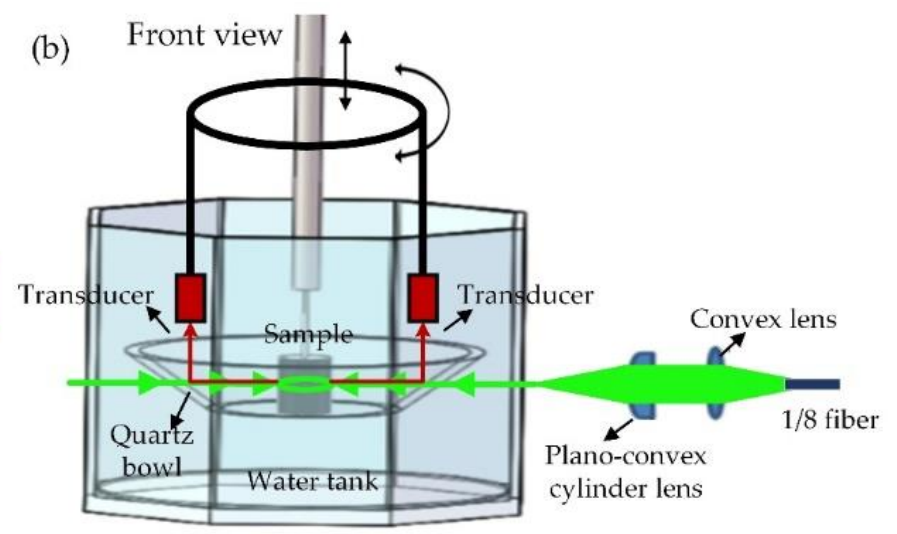

(d)

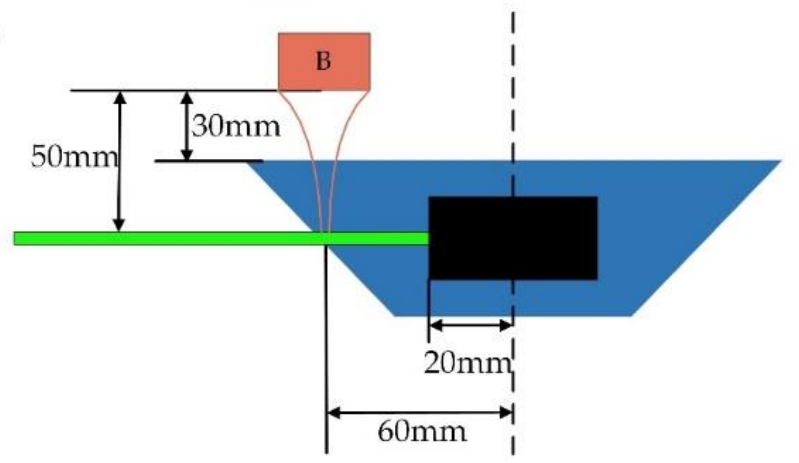

Figure 2. (a) Top view of the core optical path, (b) front view of the core optical path, (c) ultrasonic transducer and its size parameters, and (d) installation method and position diagram of ultrasonic transducer.

It is worth mentioning that this system was the first photoacoustic tomography system for innovatively using the structure of quartz bowl with the characteristics of light transmission and ultrasonic reflection for maintaining the photoacoustic coplanarity of the system while collecting photoacoustic signals. This means that the circular spot was in the same layer as the detection plane of ultrasonic transducer. The slim photoacoustic coplanar configuration eliminated noise from nearby planes, and enables optimal photoacoustic signal excitation and detection. The light transmittance and ultrasonic reflection efficiency are two critical parameters for a quartz bowl, which were $88.25 \%$ and $202.465 \%$. The signal generated by the sample was reflected by the quartz bowl and detected by the dual-foci virtual point ultrasonic transducer (Olympus, Tokyo, Japan) placed vertically above the bowl with a $5 \mathrm{MHz}$ central frequency and an $18 \mathrm{~mm}$ diameter of the detection plane, as shown in Figure 2c. The surface of the dual-foci transducer adopted the method of dual concave crafting in order to achieve foci in two perpendicular directions, respectively indicated by the letters A and B in Figure 2c. The focal length was $11.25 \mathrm{~mm}$ and the directional angle was $100^{\circ}$ in direction $\mathrm{A}$, while in direction $\mathrm{B}$, the focal length was about $90.0 \mathrm{~mm}$ and the directional angle was $11.4^{\circ}$. It can be concluded from the above parameters that the large receiving angle based on its virtual point in direction A contributed to sparse sampling, while the long focal length in direction B formed a long focal zone $(\approx 52 \mathrm{~mm})$ that determined its applicability for large imaging targets. The installation position of the ultrasonic transducer is shown in Figure 2d. In the 3D-PACT system, two custom-built virtual-point ultrasonic transducers were evenly distributed on the motor rotary table. The rotating motor performed a circular motion according to the preset speed, and the high-speed data acquisition device could complete the collection of the photoacoustic signal in the process of the motor rotation according to the timing trigger signal.

During the whole working process, the photoacoustic signals were obtained through rotating the swivel table $180^{\circ}$ driven by a rotary stepping motor and moving the sample 0.1 mm vertically at a time driven by a vertical stepper motor. After amplification (Ultrasonic Transceiver, 5073PR, Olympus, Tokyo, Japan) and digitization (Data Acquisition card, ATS330, AlazarTech, Canada), 
the raw photoacoustic data was be sent and stored in a personal computer (PC) to reconstruct the 3D photoacoustic image. The detection signal under the large receiving angle held by the virtual point-based ultrasonic transducer used in this system could provide more sample information, which contributed to recovering images with less data. In the reconstruction algorithm, the shape of the sample could be reconstructed by the improved back-projection reconstruction algorithm. The photoacoustic system adopted LabVIEW_2014 to implement the overall control system design, which realized the fully automatic man-machine interaction.

\subsection{The Reconstruction Algorithm of the 3D-PACT System}

According to the principle of photoacoustic imaging, the homogeneous wave equation can be formulized as:

$$
\left(\nabla^{2}-\frac{1}{c^{2}} \frac{\partial^{2}}{\partial t^{2}}\right) p(r \prime, t)=-p_{0}(r) \frac{d \delta(t)}{d t}
$$

where $c$ is the velocity of sound and $p$ is the initial sound pressure generated by the energy deposition of the pulsed laser. By solving the above wave equation, the photoacoustic signal detected by the ultrasonic transducer at any time $t$ and any position $r^{\prime}$ is obtained, which can be expressed as:

$$
p(r \prime, t)=\frac{\partial}{\partial t}\left[\frac{1}{4 \pi c^{3} t} \int d r \cdot p_{0}(r) \cdot \delta\left(t-\frac{|r \prime-r|}{c}\right)\right]
$$

The distribution of the original photoacoustic signal can be recovered by using the back-projection reconstruction algorithm [37-39]. Furthermore, the traditional back-projection reconstruction algorithm is shown in Equation (3):

$$
p_{0}(r)=\left.\frac{1}{4 \pi c^{3}} \int d S \frac{1}{t}\left[\frac{p(r \prime, t)}{t}-\frac{\partial p(r \prime, t)}{\partial t}\right]\right|_{t=|r \prime-r| / c}
$$

With the development of the traditional back-projection reconstruction algorithm, a reconstruction algorithm of a ring-shaped array photoacoustic image based on a sensitivity factor is proposed in this study. The photoacoustic signals collected by the transducer were back-projected to each pixel on the arc established by time in the imaging area, which is illustrated in Figure 3a as a schematic diagram. The aforementioned virtual point ultrasonic transducer has a large signal receiving angle $\left(100^{\circ}\right.$, corresponding to the angle $\Phi$ in the figure), and the transducer is relatively sensitive to the central region of its receiving scope. Therefore, a variable sensitivity factor $S(\psi)$ was introduced in the algorithm of image reconstruction, and it decreased with the increase of $\psi$, which is the angle between the connecting line of the pixel and probe and the center line of the detection area. In the process of algorithm implementation, the basic Equation (4) needed to be discretized first:

$$
p_{0}(r)=\left.\sum_{i=1}^{n}\left[p\left(r^{\prime \prime}, t\right)-t \frac{\partial p(r \prime, t)}{\partial t}\right]\right|_{t=\left|r^{\prime \prime}-r\right| / c}
$$

where $r^{\prime \prime}$ is a distance parameter introduced in the process of discretization. Taking the angle of the receiving signal of the ultrasound probe into account, the back-projection reconstruction algorithm based on sensitivity factor is proposed and can be expressed as:

$$
p_{0}(r)=\left.\sum_{i=1}^{n} S(\psi)_{(r, i)}\left[p\left(r^{\prime \prime}, t\right)-t \frac{\partial p(r \prime, t)}{\partial t}\right]\right|_{t=\left|r^{\prime \prime}-r\right| / c}
$$



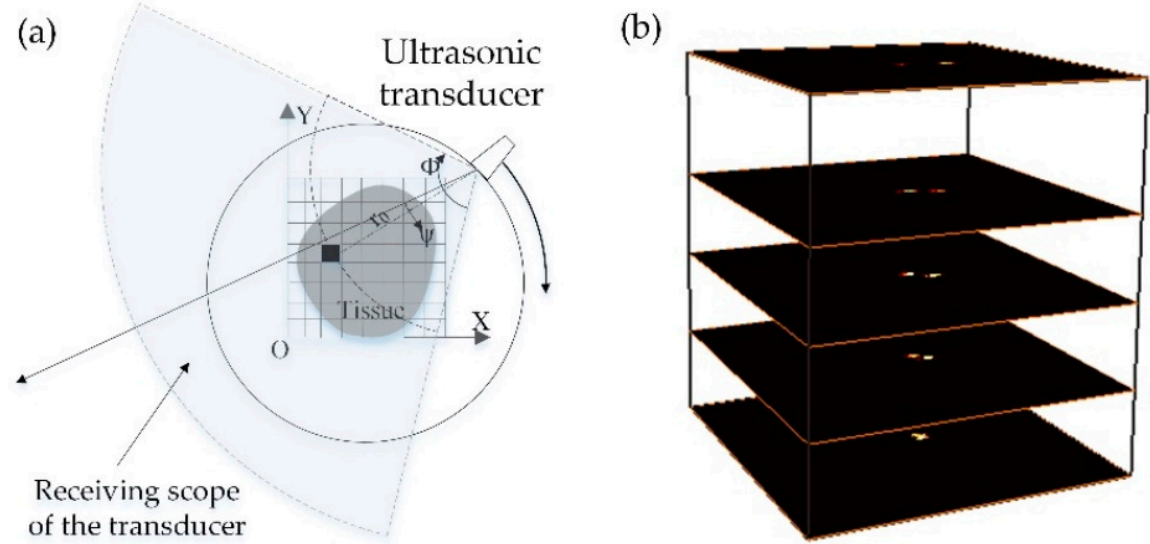

Figure 3. (a) A 2D schematic of the improved back-projection reconstruction algorithm, and (b) 3D photoacoustic image reconstruction.

In order to perform the 3D image rendering, a large number of B-scans were loaded into Amira 5.4.3 to be superimposed and processed, as shown in Figure 3b.

\section{Results}

\subsection{Performance Test of the $3 D-P A C T$}

\subsubsection{Feasibility Verification of Quartz Bowl in the System}

First of all, the photoacoustic experiments were carried out in the ultrasonic transmission mode and ultrasonic reflection mode to verify the feasibility of the quartz bowl. The schematic diagrams of the two experimental devices are shown in Figure $4 a, b$, respectively. Black tape was selected as the target due to the strong laser absorption. The energy at the fiber exit was around $100 \mu \mathrm{J}$. The energy $\mathrm{E}$ in Table 1 refers to the laser energy of the imaging target surface.
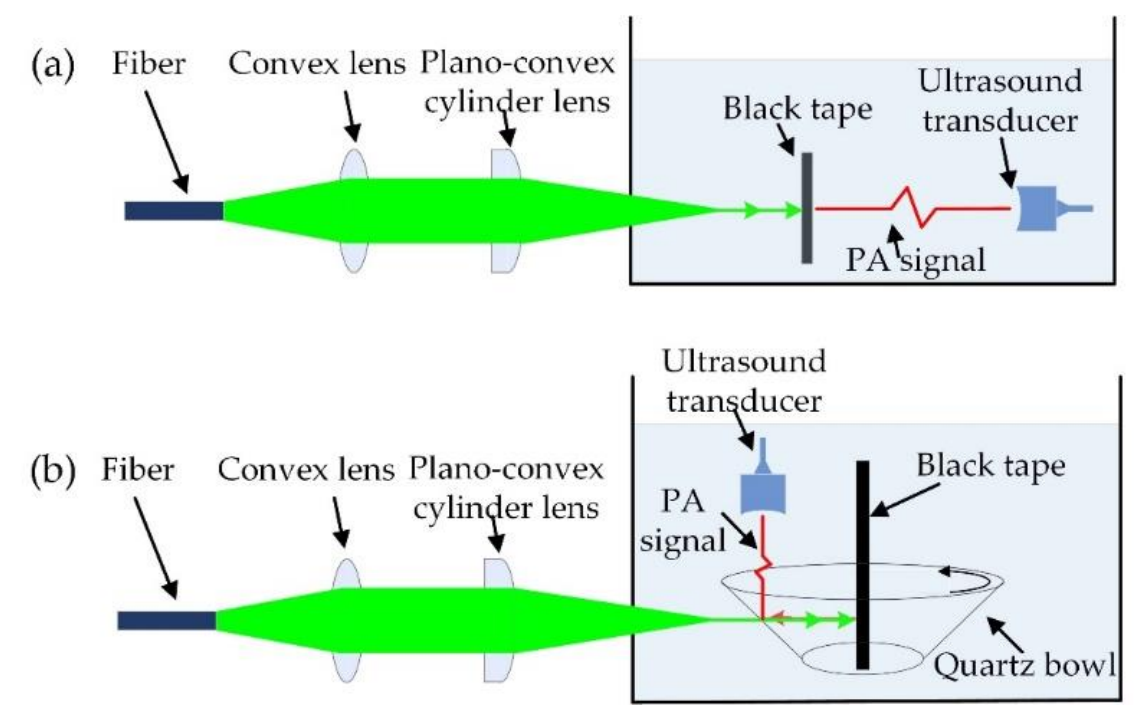

Figure 4. (a) Schematic diagram of photoacoustic imaging device in ultrasonic transmission mode.

(b) Schematic diagram of photoacoustic imaging device in ultrasonic reflection mode.

The ultrasonic reflection efficiency $H$ of the quartz bowl is defined as the ratio of the photoacoustic signal amplitude in the above two signal acceptance modes in the research. The specific expression is:

$$
H=G_{r e f} / G_{t r m}
$$


where $G_{r e f}$ is the signal amplitude per microjoule laser energy in the ultrasonic reflection mode and $G_{t r m}$ is the signal amplitude per microjoule laser energy in the ultrasonic transmission mode. Furthermore, $G_{t r m}$ was about $0.01232 \mathrm{~V} / \mu \mathrm{J}$, found by analyzing the photoacoustic signal obtained from photoacoustic experiment in ultrasonic transmission mode. The quartz bowl was divided into eight areas $\left(\mathrm{Q}_{1}-\mathrm{Q}_{8}\right)$, and the quartz bowl's ultrasonic reflection efficiency was tested after subtracting the light attenuation of each area.

Table 1. The ultrasonic reflection efficiency of the quartz bowl.

\begin{tabular}{ccccc}
\hline Areas & $\mathbf{E}(\mu \mathrm{J})$ & $\begin{array}{c}\text { Mean of Signal } \\
\text { Amplitude } \mathbf{P}(\mathrm{V})\end{array}$ & $\mathrm{G}_{\text {ref }}=\mathbf{P} / \mathbf{E}(\mathrm{V} / \mu \mathrm{J})$ & $\mathbf{H}=\mathrm{G}_{\text {ref }} / \mathrm{G}_{\text {trm }}$ \\
\hline $\mathrm{Q}_{1}$ & 88.7614 & 2.236 & 0.02519 & $204.46 \%$ \\
$\mathrm{Q}_{2}$ & 88.3206 & 2.214 & 0.02507 & $203.49 \%$ \\
$\mathrm{Q}_{3}$ & 88.0611 & 2.183 & 0.02480 & $201.30 \%$ \\
$\mathrm{Q}_{4}$ & 88.1574 & 2.195 & 0.02490 & $202.11 \%$ \\
$\mathrm{Q}_{5}$ & 88.2163 & 2.222 & 0.02520 & $204.54 \%$ \\
$\mathrm{Q}_{6}$ & 88.0742 & 2.156 & 0.02448 & $198.70 \%$ \\
$\mathrm{Q}_{7}$ & 88.0688 & 2.174 & 0.02469 & $200.41 \%$ \\
$\mathrm{Q}_{8}$ & 88.3672 & 2.229 & 0.02522 & $204.71 \%$ \\
\hline
\end{tabular}

The ultrasonic reflection efficiency of quartz bowl was between $198.70 \%$ and $204.71 \%$, which could be seen by noting that the amplitude of the photoacoustic signal was nearly doubled in ultrasonic reflection mode. Experiments showed that the quartz bowl used in the system had a higher efficiency of ultrasonic signal reflection, which was helpful for the collection of photoacoustic signals in PACT.

\subsubsection{Resolution Test of the System}

In the resolution test experiment, a black sphere with a diameter of $0.3 \mathrm{~mm}$ in $2 \%$ agar was used as an imaging target and photoacoustic signal source, and was placed in the center of the circular illumination zone, as shown in Figure 5a. Because of its all-black color, the sphere was a good absorber at the laser wavelength of $532 \mathrm{~nm}$ used in the system. In the process of data acquisition, the sampling rate was $50 \mathrm{MHz}$, the number of sampling points in a B-scan was 180 and the total laser energy reaching the surface of the phantom was about $3.2 \mathrm{~mJ}$ (the optical fluence of each bundle was $0.7 \mathrm{~mJ} / \mathrm{mm}^{2}$ ). During the experiment, the imaging target, quartz bowl, and the ultrasonic transducer were all immersed in distilled water to achieve acoustic coupling. The reconstructed photoacoustic image is shown in Figure 5b. It should be noted that the imaging resolution in this research was defined as the full width at half maximum of the normalized absorption intensity at the centerline of photoacoustic image Figure $5 b$. As shown in Figure $5 c$, the imaging resolution of the system was $0.31 \pm 0.02 \mathrm{~mm}$ based on quantitative calculations. In addition, according to the raw data analysis of the photoacoustic signal shown in Figure $5 \mathrm{~d}$, it can be seen that the intensity of the photoacoustic signal of the imaging target was relatively uniform at the cross section, and there was no phenomenon showing that the photoacoustic signal was submerged. These also reflected the ability of the system to maintain the photoacoustic coplanar property.
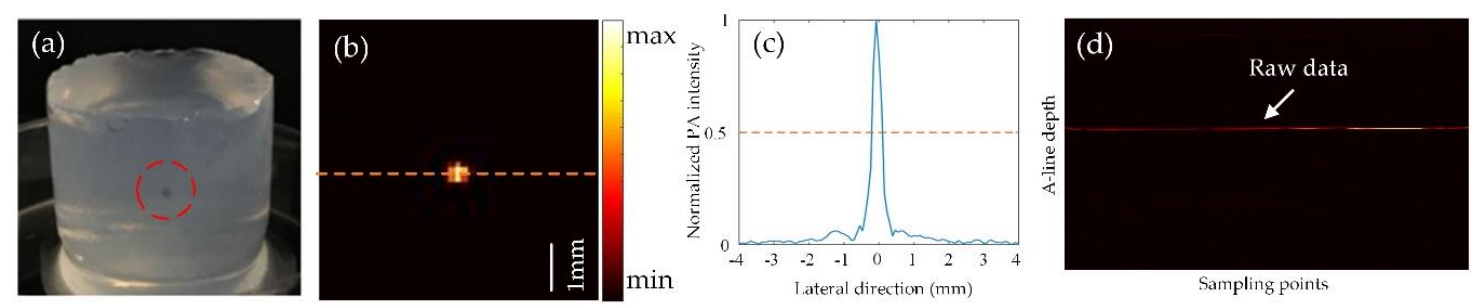

Figure 5. (a) Phantom of resolution test, (b) reconstructed PA image of target, (c) the profile along the dotted line in (b), and (d) raw data of a B-scan. 


\subsubsection{The Superiority of Photoacoustic Coplanar Configuration and Virtual Point Detection}

In the photoacoustic tomography system proposed herein, the narrow sound beam of dual-foci virtual point ultrasonic transducer and the annular illumination beam were coupled to form a photoacoustic coplanar mode based on the quartz bowl with the characteristics of laser transmission and ultrasonic reflection. To prove the advantages of the virtual-point ultrasonic detection and photoacoustic coplanar mode, a comparative experiment was performed using a conventional unfocused ultrasonic transducer (V310, Olympus, Tokyo, Japan). The results of the comparative experiment are shown in Figure 6 . In the experiment, the black tape was evenly wrapped around a steel column with a diameter of $25 \mathrm{~mm}$ as an imaging phantom, as shown in Figure 6a. The number of sampling points per imaging section was 90 , and it took about $9 \mathrm{~s}$ to complete a photoacoustic data acquisition of a cross section. Figure $6 c$,d showed the reconstruction images from the dual-foci virtual point ultrasonic transducer and unfocused ultrasonic transducer, respectively. From a qualitative point of view, it is obvious that the quality of the reconstructed image with the dual-foci transducer was better than its counterpart in terms of qualities such as shape-similarity and image-purity.
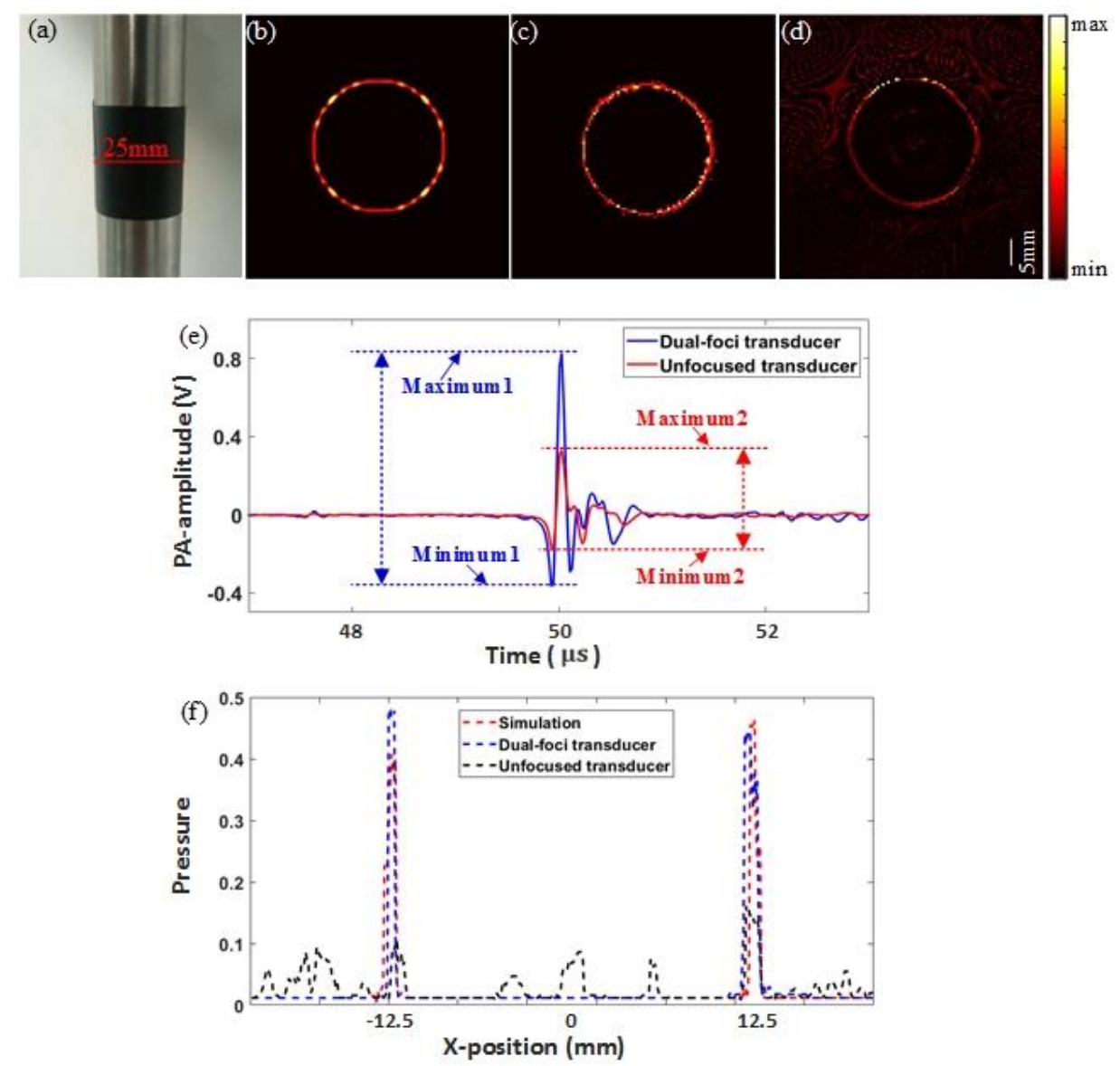

Figure 6. (a) Phantom of the comparative experiment. (b) Reference standard image by K-Wave simulation. Reconstructed PA images obtained with (c) the dual-foci transducer and (d) the traditional unfocused transducer. (e) A-Line plots from dual-foci transducer and traditional unfocused transducer. (f) Absorption intensity distribution at the centerline of $(\mathbf{a}-\mathbf{c})$.

In order to quantitatively analyze the reconstruction quality of Figure $6 c, d$, the simulation result of the imaging target (shown in Figure $6 \mathrm{~b}$ ) was used as a reference standard. The photoacoustic raw data of Figure $6 \mathrm{~b}$ was obtained using a K-wave simulation, and then reconstructed using the improved back projection algorithm mentioned above. The performance indicators, such as MSE (mean squared 
error), PSNR (peak signal to noise ratio), and SSIM (structural similarity) [40-42] were calculated separately according to the following formulae. The MSE index is given as:

$$
M S E=\frac{1}{m n} \sum_{i=1}^{m} \sum_{j=1}^{n}\|I(i, j)-K(i, j)\|^{2}
$$

The $I(i, j)$ and $K(i, j)$ in the formula represent the reconstructed image and the reference standard image, which refer to the reconstructed photoacoustic image and the K-Wave simulation image in this paper, and the parameters $m$ and $n$ represent the length and width of the image.

PSNR is defined as:

$$
P S N R=20 * \log _{10}\left(\frac{M A X_{1}}{\sqrt{M S E}}\right)
$$

where $M A X_{1}$ is the gray level of the image. Lastly, the SSIM is given as:

$$
\operatorname{SSIM}(x, y)=\frac{\left(2 \mu_{x} \mu_{y}+c_{1}\right)\left(2 \sigma_{x y}+c_{2}\right)}{\left(\mu_{x}^{2}+\mu_{y}^{2}+c_{1}\right)\left(\sigma_{x}^{2}+\sigma_{y}^{2}+c_{2}\right)}
$$

where $x$ and $y$ represent the two images for comparison, $\mu_{x}$ and $\mu_{y}$ are the mean of the image, $\sigma_{x}$ and $\sigma_{y}$ represent the variance of image, $\sigma_{x y}$ is the covariance of $x$ and $y, c_{1}=\left(k_{1} L\right)^{2}$ and $c_{2}=\left(k_{2} L\right)^{2}$ are two constants used to avoid division by zero in the formula, $L=2^{B}-1$ is the range of pixel values, and $k_{1}=0.01, k_{2}=0.03$ are default values.

The results of the above three performance indexes are shown in the following Table 2.

Table 2. Quality evaluation of reconstructed images.

\begin{tabular}{cccc}
\hline Index & PSNR (dB) & MSE & SSIM \\
\hline PA image (c) (Dual-foci transducer) & 35.9379 & 0.0051 & 0.9995 \\
PA image (d) (Unfocused transducer) & 15.2504 & 0.0350 & 0.9174 \\
\hline
\end{tabular}

It can be seen form Table 2 that the performance indicators SSIM and PSNR of the reconstructed images were greatly improved in the case of virtual-point ultrasound detection, while the value of MSE was obviously reduced. The three performance indicators of the reconstructed photoacoustic image under the dual-foci virtual point transducer were better than those under the unfocused transducer that verified the reliability of the proposed system.

The PSNR of the photoacoustic signal from the dual-foci virtual point ultrasound transducer was $35.9379 \mathrm{~dB}$, which was much larger than that of the conventional unfocused transducer (15.2504 dB), indicating that the dual-foci virtual point transducer had a higher detection sensitivity. Moreover, the high detection sensitivity of the photoacoustic tomography system is further illustrated in Figure 6e, in which the blue and red A-line plots corresponding to the raw photoacoustic signal with the dual-foci virtual-point ultrasonic transducer and the traditional unfocused ultrasonic transducer are depicted, respectively. The two A-line plots were randomly obtained under the premise of the same scanning position and sampling points. It can be seen that the photoacoustic signal from the dual-foci ultrasonic transducer had an amplitude increase of nearly 2.5-fold, which greatly improved the signal to noise ratio of the photoacoustic image. Figure $6 \mathrm{f}$ shows the absorption intensity at the position of the centerline of the reconstructed image, and it can be found that the absorption intensity of the center line position of the photoacoustic image obtained by the unfocused ultrasonic transducer was weak (corresponding to the black dotted line in the Figure 6f), and there are signs that the signal was flooded by noise.

In addition, the virtual-point ultrasonic transducer has a large receiving angle, which makes the photoacoustic tomography under sparse sampling possible. In the experiment mentioned above, although the number of sampling points per cross section was 90 , the system with virtual-point 
detection could still perform high quality photoacoustic tomography. However, there were some artifacts in the photoacoustic image under the traditional unfocused transducer. The experiment proved that the system proposed in this paper can improve the imaging speed and reduce the burden of data acquisition device using sparse sampling. The comparative experiments analyzed the photoacoustic signal acquisition mode based on photoacoustic coplanar structure and virtual-point ultrasound detection from a quantitative point of view.

\subsubsection{Verification Experiment on Large-Scale Imaging}

In order to verify the large-scale imaging characteristics of the system, a photoacoustic tomography experiment was performed using a phantom (a hollow cylinder wrapped with black tape, the diameter is $52 \mathrm{~mm}$ ), as shown in Figure $7 \mathrm{a}$. Figure $7 \mathrm{~b}$ shows a photoacoustic image of a cross section. It can be seen that the imaging range of the system could be up to $52 \mathrm{~mm}$ in diameter.
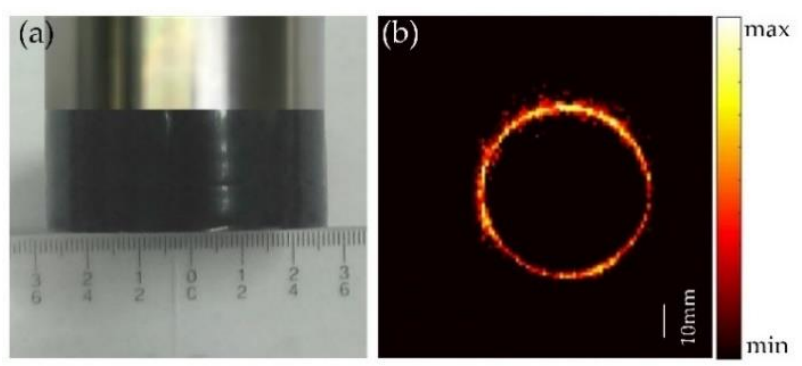

Figure 7. (a) Phantom of imaging range test, (b) Reconstructed PA image.

To show the 3D photoacoustic imaging in a wide area, four black polyethylene tubes with large spatial distances were designed as imaging targets, as shown in Figure 8a. Figure $8 \mathrm{~b}$ is a reconstructed photoacoustic image of the scanning section indicated by the dotted line in the real figure. Figure $8 \mathrm{c}$ is one of an A-line signal of the B-scan, in which the four signal peaks correspond to the four polyethylene tubes respectively, and the distance between the signal peaks matches the distance between actual tubes. Figure $8 \mathrm{~d}$ is a 3D photoacoustic image of the imaging target, and the quantized spatial distance is consistent with those shown in Figure 8a,b. It can be seen that the 3D photoacoustic image could more accurately reflect the information of the phantom, such as shape, size, deformation, and so on. According to the photoacoustic images of this test experiment, it can be concluded that even if there was a large space distance, the target shown in Figure 8a could still be clearly imaged.
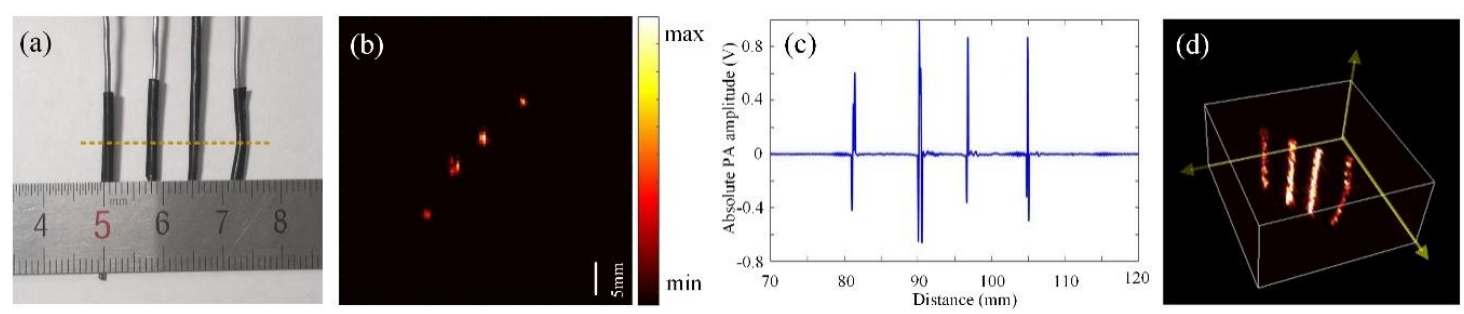

Figure 8. (a) Phantom of confirmatory experiment on scope, (b) reconstructed PA image of target,

(c) one of an A-line of (b), and (d) 3D photoacoustic image.

\subsection{Photoacoustic Experiments of Different Kinds of Phantoms}

After completing the test experiment of resolution and the verification experiment of a large imaging range, a 3D photoacoustic imaging experiment of the vascular phantom was performed to further verify the feasibility of the imaging system. Two black wires with a diameter of $1 \mathrm{~mm}$ were used to make a phantom that simulates blood vessels in tissue, as shown in Figure 9a. In this experiment, photoacoustic signals from 180 positions were acquired on each imaging section, that is, a photoacoustic signal was collected for every $2^{\circ}$ of rotation of the ultrasonic probe; it took about $18 \mathrm{~s}$ 
to acquire the information of a B-scan. The step size of the vertical movement of the phantom was $0.2 \mathrm{~mm}$, and $120 \mathrm{~B}$-scans were scanned during the experiment. Figure $9 \mathrm{~b}$ shows the photoacoustic images of three B-scans and the corresponding raw data. It can be seen that the system can accurately reflect the shape and size of the phantom. Figure $9 \mathrm{c}$ shows the three-dimensional photoacoustic image of the phantom, which not only clearly identifies the different parts of the phantom, but also reflects the change of it in the vertical direction, that is, the reconstructed result is highly consistent with the blood vessel phantom. This result indicates that the 3D-PACT is capable of 3D imaging small objects of different spatial distribution and orientation in high quality.
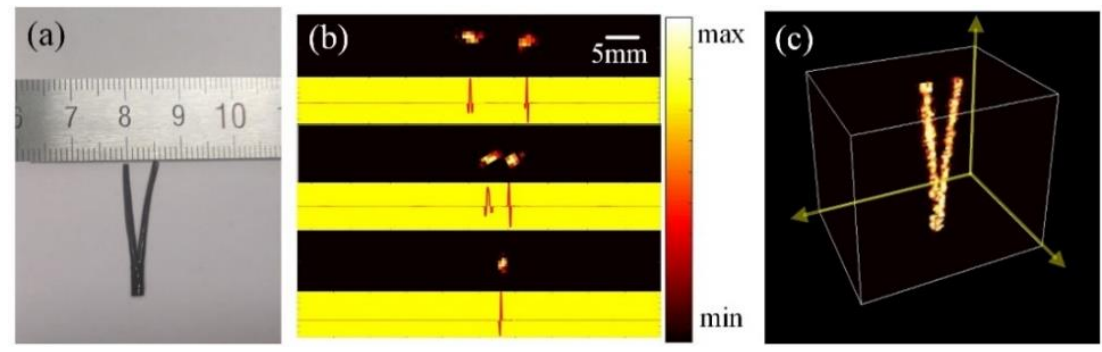

Figure 9. (a) Photograph of a blood vessel phantom, (b) PA images and A-lines of several B-scans, and (c) $3 \mathrm{D}$ photoacoustic image.

Subsequently, a 3D photoacoustic experiment was performed on a tumor phantom obtained by soaking the plasticine, whose dimensions are shown in Figure 10a, in black ink. In this experiment, a total of 90 images were obtained by scanning the phantom with a vertical interval of $0.1 \mathrm{~mm}$, and the energy of the pulsed laser reaching the phantom surface was approximately $3.6 \mathrm{~mJ}$. It can be seen that the laser could penetrate the imaging phantom and the laser absorption characteristics of each B-scan, and each point on it could be well-reflected from both two-dimensional and three-dimensional photoacoustic images demonstrated in Figure 10b,c separately. By comparing Figure 10a,c, it can be seen that the size of the absorption region on the photoacoustic image was substantially the same as the actual size of the corresponding section, which verified the feasibility and reliability of this system by showing that the shape and size of tumor analogs can be well-reflected through 3D photoacoustic imaging.
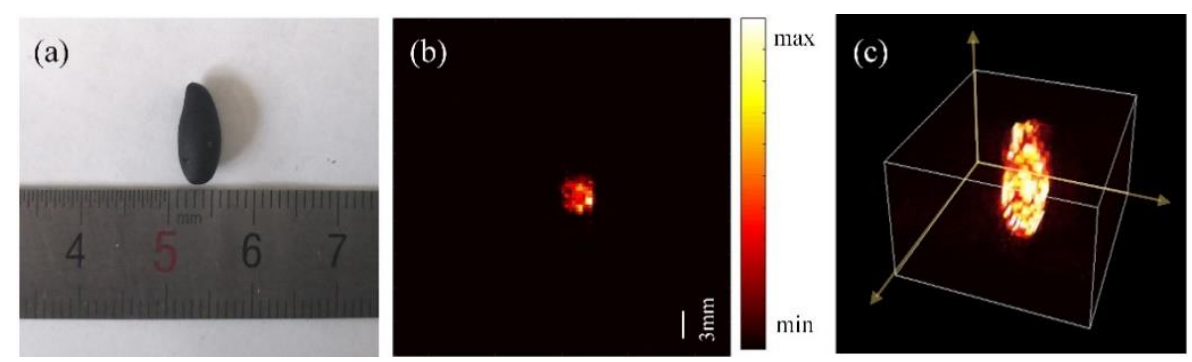

Figure 10. (a) Photograph of a tumor phantom, (b) PA image of a B-scan, and (c) three-dimensional photoacoustic image.

Finally, in order to verify the photoacoustic imaging of some irregularly shaped tissues, 3D photoacoustic experiments were carried out on complex-shaped phantoms. For example, a phantom made of knotted black wire with a diameter of about $0.6 \mathrm{~mm}$ is shown in Figure 11a. The sampling frequency, sampling position, laser energy, and z-axis spacing of this experiment were the same as those above. Figure $11 \mathrm{~b}$ is a $3 \mathrm{D}$ photoacoustic image reconstructed by using the detected photoacoustic signals detected from $90 \mathrm{~B}$-scans spaced $0.1 \mathrm{~mm}$ apart, indicating that the reconstructed photoacoustic image could present the actual shape and dimensions of the target clearly and with high quality. 


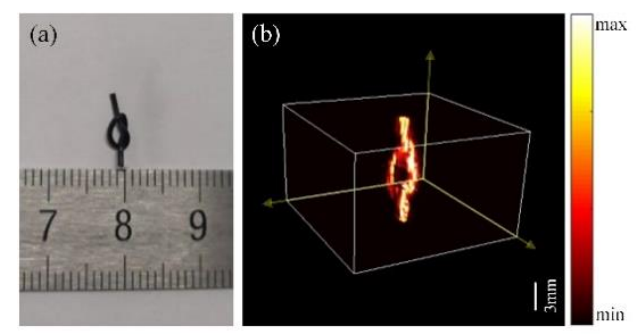

Figure 11. (a) Photograph of a knot phantom and (b) 3D photoacoustic image.

\section{Discussion and Conclusions}

A 3D-PACT imaging system based on full-view illumination and detection was proposed and developed in this study. In the design of optical path, the system adopted a mode of circular illumination with the even arrangement of eight laser beams around the water tank to ensure that the imaging target received more uniform laser radiation, which improved the low-efficiency of photoacoustic imaging whose illumination was from the top to the bottom. Second, drawing on the design of photoacoustic coaxial confocal in photoacoustic microscopy imaging technology, a photoacoustic coplanar structure was proposed by means of a quartz bowl with light-transmitting and ultrasound-reflecting properties. The system extended the photoacoustic coupling from a single dimension to a two-dimensional space, increasing the photoacoustic detection range of the imaging section and improving the signal to noise ratio of the photoacoustic signal. In addition, the dual-foci virtual point ultrasonic transducer with a large receiving angle improved the imaging speed of the system via sparse sampling. In the reconstruction method of the photoacoustic image, taking the receiving angle of ultrasound transducer into account, the improved back-projection reconstruction algorithm based on sensitivity factor was achieved, and the photoacoustic image reconstruction using the algorithm took $1.4287 \mathrm{~s}$.

The practicality of the illumination method and the designed structure enable the system to perform clear and high-quality 3D photoacoustic imaging on targets with complex surfaces or requiring a large imaging range. The feasibility of the system was verified through systematic testing of resolution, imaging range, and photoacoustic coplanar property and 3D photoacoustic experiments on various phantoms. The best compromise was reached between imaging performance and system cost.

The imaging speed of the system was about $9 \mathrm{~s}$ in one cross section (90 sampling points per B-scan, it took about 15 min to scan 100 B-scans), and had not reached the requirements of fast imaging; however, the phantom experiments conducted in this study have demonstrated the characteristics of the system. The next step will be focused on the research of sparse circular array ultrasonic transducer based on virtual point and resolution improvement. In practical applications, the system is expected to be used for corresponding work in the pathological study of an in vitro tumor and the research of targeted photoacoustic probes for breast cancer cells. Moreover, the 3D reconstruction will be further investigated.

Author Contributions: Design and construction of the 3D-PACT, M.S., D.H., and L.M.; Completion of photoacoustic imaging experiments, D.H., W.Z., and Y.Q.; Control system design, M.S., D.H., and L.M.; System debugging and improvement, Y.L., Y.Q., and W.Z.; Algorithm implementation, M.S., D.H., and Y.L.; Paper writing, D.H., W.Z., and M.S.

Funding: This research was funded by the National Key R \& D Program of China (Grant No. 2017YFE0121000 and No. 2018YFC0114800), National Natural Science Foundation of China (Grant No. 11574064 and No. 11874133), Shandong Provincial Natural Science Foundation, China (Grant No. ZR2017MF041 and ZR2018MF026), the Science and Technology Development Plan Project of Shandong Province (Grant No. 2018GGX103047 and 2016GGX103032), and the Development Plan of Chinese Academy of Sciences and Wego Group (Grant No. 2017011).

Acknowledgments: This work was partly supported by the Institute of Biomedical and Health Engineering at Shen Zhen Institute of Advanced Technology, Chinese Academy of Sciences (SIAT).

Conflicts of Interest: The authors declare no conflict of interest. 


\section{References}

1. Xu, M.; Wang, L.V. Photoacoustic imaging in biomedicine. Rev. Sci. Instrum. 2006, 77, 41101. [CrossRef]

2. Wang, L.V.; Hu, S. Photoacoustic Tomography: In Vivo Imaging from Organelles to Organs. Science 2012, 335, 1458-1462. [CrossRef]

3. Beard, P. Biomedical photoacoustic imaging. Interface Focus 2011, 1, 602. [CrossRef]

4. Wang, L.V.; Wu, H. Biomedical optics: Principles and imaging. J. Biomed. Opt. 2008, 13, 049902. [CrossRef]

5. Liu, T.; Sun, M.; Feng, N.; Wu, Z.; Shen, Y. Multiscale Hessian filter-based segmentation and quantification method for photoacoustic microangiography. Chin. Opt. Lett. 2015, 13, 091701.

6. Lin, X.; Sun, M.; Feng, N.; Hu, D.; Shen, Y. Monte Carlo light transport-based blood vessel quantification using linear array photoacoustic tomography. Chin. Opt. Lett. 2017, 15, 111701.

7. Wang, X.; Pang, Y.; Ku, G.; Xie, X.; Stoica, G.; Wang, L.V. Noninvasive laser-induced photoacoustic tomography for structural and functional in vivo imaging of the brain. Nat. Biotechnol. 2003, 21, 803-806. [CrossRef]

8. Wang, L.V.; Yao, J. A practical guide to photoacoustic tomography in the life sciences. Nat. Methods 2016, 13, 627-638. [CrossRef]

9. Kruger, R.A.; Lam, R.B.; Reinecke, D.R.; Rio, S.P. Photoacoustic angiography of the breast. Med. Phys. 2010, 37, 6096-6100. [CrossRef]

10. Wang, S.; Lin, J.; Wang, T.; Chen, X.; Huang, P. Recent Advances in Photoacoustic Imaging for Deep-Tissue Biomedical Applications. Theranostics 2016, 6, 2394-2413. [CrossRef]

11. Wang, H.; Liu, C.; Gong, X.; Hu, D.; Lin, R.; Sheng, Z.; Zheng, C.; Yan, M.; Chen, J.; Cai, L.; et al. In vivo photoacoustic molecular imaging of breast carcinoma with folate receptor-targeted indocyanine green nanoprobes. Nanoscale 2014, 6, 14270-14279. [CrossRef]

12. Cao, M.; Yuan, J.; Du, S.; Xu, G.; Wang, X.; PL, C.; Liu, X. Full-view photoacoustic tomography using asymmetric distributed sensors optimized with compressed sensing method. Biomed. Signal Process. Control 2015, 21, 19-25. [CrossRef]

13. Wang, X.; Roberts, W.W.; Carson, P.L.; Wood, D.P.; Fowlkes, J.B. Photoacoustic tomography: A potential new tool for prostate cancer. Biomed. Opt. Express 2010, 1, 1117. [CrossRef]

14. Wurzinger, G.; Nuster, R.; Schmitner, N.; Gratt, S.; Meyer, D.; Paltauf, G. Simultaneous three-dimensional photoacoustic and laser-ultrasound tomography. Biomed. Opt. Express 2013, 4, 1380. [CrossRef]

15. Xie, Z.; Tian, C.; Chen, S.L.; Ling, T. 3D high resolution photoacoustic imaging based on pure optical photoacoustic microscopy with microring resonator. In Proceedings of the SPIE 8943, Photons Plus Ultrasound: Imaging and Sensing 2014; International Society for Optics and Photonics: Bellingham, WA, USA, 2014.

16. Moradi, H.; Honarvar, M.; Tang, S.; Salcudean, S.E. Iterative photoacoustic image reconstruction for three-dimensional imaging by conventional linear-array detection with sparsity regularization. In Proceedings Volume 10064, Photons Plus Ultrasound: Imaging and Sensing; International Society for Optics and Photonics: Bellingham, WA, USA, 2017.

17. Tan, Y.; Xia, K.; Ren, Q.; Li, C. Three-dimensional photoacoustic imaging via scanning a one dimensional linear unfocused ultrasound array. Opt. Express 2017, 25, 8022-8028. [CrossRef]

18. Vaithilingam, S.; Ma, T.J.; Furukawa, Y.; Wygant, I.O.; Zhuang, X.; De La Zerda, A.; Oralkan, O.; Kamaya, Y.; Gambhir, S.S.; Jeffrey, R.B.; et al. Three-dimensional photoacoustic imaging using a two-dimensional CMUT array. IEEE Trans. Ultrason. Ferroelectr. Freq. Control 2009, 56, 2411-2419. [CrossRef]

19. Paltauf, G.; Nuster, R.; Burgholzer, P.; Haltmeier, M. Three-dimensional photoacoustic tomography using acoustic line detectors. Proc. SPIE 2007, 6437,64370N.

20. Ermolayev, V.; Dean-Ben, X.L.; Mandal, S.; Ntziachristos, V.; Razansky, D. Simultaneous visualization of tumour oxygenation, neovascularization and contrast agent perfusion by real-time three-dimensional optoacoustic tomography. Eur. Radiol. 2016, 26, 1843-1851. [CrossRef]

21. Wang, B.; Xiang, L.; Jiang, M.S.; Yang, J.; Zhang, Q.; Carney, P.R.; Jiang, H. Photoacoustic tomography system for noninvasive real-time three-dimensional imaging of epilepsy. Biomed. Opt. Express 2012, 3, 1427-1432. [CrossRef]

22. Hui, L.; Yu, C.; Hongbo, L.; Dong, P.; Yukun, Z.; Kun, W.; Jie, T. High-speed and 128-channel multi-spectral photoacoustic tomography system for small animal. Laser Technol. 2017, 41, 669-674.

23. Razansky, D.; Buehler, A.; Ntziachristos, V. Volumetric real-time multispectral optoacoustic tomography of biomarkers. Nat. Protoc. 2011, 6, 1121-1129. [CrossRef] 
24. Xia, J.; Chatni, M.R.; Maslov, K.; Guo, Z.; Wang, K.; Anastasio, M.; Wang, L.V. Whole-body ring-shaped confocal photoacoustic computed tomography of small animals in vivo. J. Biomed. Opt. 2012, 17, 050506. [CrossRef]

25. Wong, T.T.; Zhang, R.; Zhang, C.; Hsu, H.C.; Maslov, K.I.; Wang, L.; Shi, J.; Chen, R.; Shung, K.K.; Zhou, Q.; et al. Label-free automated three-dimensional imaging of whole organs by microtomy-assisted photoacoustic microscopy. Nat. Commun. 2017, 8, 1386. [CrossRef]

26. Paltauf, G.; Hartmair, P.; Kovachev, G.; Kovachec, G.; Nuster, R. Piezoelectric line detector array for photoacoustic tomography. Photoacoustics 2017, 8, 28-36. [CrossRef]

27. Li, C.; Aguirre, A.; Gamelin, J.; Maurudis, A.; Zhou, Q.; Wang, L.V. Real-time photoacoustic tomography of cortical hemodynamics in small animals. J. Biomed. Opt. 2010, 15, 010509. [CrossRef]

28. Xia, J.; Chatni, M.; Maslov, K.; Wang, L.V. Anatomical and metabolic small-animal whole-body imaging using ring-shaped confocal photoacoustic computed tomography. In Proceedings of the Photons Plus Ultrasound: Imaging $\mathcal{E}$ Sensing; International Society for Optics and Photonics: Bellingham, WA, USA, 2013.

29. Ku, G.; Wang, X.; Stoica, G.; Wang, L.V. Multiple-bandwidth photoacoustic tomography. Phys. Med. Biol. 2004, 49, 1329-1338. [CrossRef]

30. Kruger, R.A.; Liu, P.Y.; Fang, Y.R.; Appledorn, C.R. Photoacoustic ultrasound (PAUS)—Reconstruction tomography. Med. Phys. 1995, 22, 1605-1609. [CrossRef]

31. Ma, R.; Taruttis, A.; Ntziachristos, V.; Razansky, D. Multispectral optoacoustic tomography (MSOT) scanner for whole-body small animal imaging. Opt. Express 2009, 17, 21414-21426. [CrossRef]

32. Pramanik, M.; Ku, G.; Wang, L.V. Tangential resolution improvement in thermoacoustic and photoacoustic tomography using a negative acoustic lens. J. Biomed. Opt. 2009, 14, 024028. [CrossRef]

33. Li, C.; Ku, G.; Wang, L.V. Negative lens concept for photoacoustic tomography. Phys. Rev. E 2008, 78, 021901. [CrossRef]

34. Perekatova, V.V.; Kirillin, M.Y.; Turchin, I.V.; Subochev, P.V. Combination of virtual point detector concept and fluence compensation in acoustic resolution photoacoustic microscopy. In Proceedings of the the 6th International Symposium on Topical Problems of Biophotonics (TPB), St Petersburg, Russia, 28 July-3 August 2017.

35. Nie, L.; Guo, Z.; Wang, L.V. Photoacoustic tomography of monkey brain using virtual point ultrasonic transducers. J. Biomed. Opt. 2011, 16, 076005. [CrossRef]

36. Zhang, H.F.; Menglin, L.; Konstantin, M.; Stocia, G.; Wang, L.V. Three-dimensional photoacoustic imaging of subcutaneous microvasculature in vivo. Proc. SPIE 2006, 6086, 60861E.

37. Minghua, X.; Wang, L.V. Universal back-projection algorithm for photoacoustic computed tomography. Phys. Rev. E Stat. Nonlinear Soft Matter Phys. 2005, 711 Pt 2, 016706.

38. Xu, M.; Wang, L.V. Pulsed-microwave-induced thermoacoustic tomography: Filtered backprojection in a circular measurement configuration. Med. Phys. 2002, 29, 1661-1669. [CrossRef] [PubMed]

39. Duoduo, H.; Zheng, S.; Yuan, Y. Reconstruction of Intravascular Photoacoustic Images Based on Filtered Back-projection Algorithm. Chin. J. Biomed. Eng. 2016, 35, 9-19.

40. Wang, Z.; Simoncelli, E.P.; Bovik, A.C. Multi-scale structural similarity for image quality assessment. In Proceedings of the IEEE Asilomar Conference on Signals, Pacific Grove, CA, USA, 9-12 November 2003; Volume 2, pp. 1398-1402.

41. Mandal, S.; Sudarshan, V.P.; Nagaraj, Y.; Dean-Ben, X.L.; Razansky, D. Multiscale edge detection and parametric shape modeling for boundary delineation in optoacoustic images. In Proceedings of the 2015 37th Annual International Conference of the IEEE Engineering in Medicine and Biology Society (EMBC) 2015, Milan, Italy, 25-29 August 2015; pp. 707-710.

42. Kazakeviciute, A.; Ho CJ, H.; Olivo, M. Multispectral Photoacoustic Imaging Artifact Removal and Denoising Using Time Series Model-Based Spectral Noise Estimation. IEEE Trans. Med. Imaging 2016, 35, 2151-2163. [CrossRef]

(C) 2019 by the authors. Licensee MDPI, Basel, Switzerland. This article is an open access article distributed under the terms and conditions of the Creative Commons Attribution (CC BY) license (http://creativecommons.org/licenses/by/4.0/). 